Journal of Business \& Management (COES\&RJ-JBM)

ISSN (E): 2306-7179 ISSN (P): 2306-8043

Publisher: Centre of Excellence for Scientific \& Research Journalism, COES\&RJ LLC

Online Publication Date \& Issue: $1^{\text {st }}$ January 2016, Vol.4, No.1, January 2016

http://centreofexcellence.net/J/JBM/JBM\%20Mainpage.htm

\title{
The Role of Information Technology in motivating students to accept e-learning adoption in universities: A case study in Jordanian universities
}

Dr. Dmaithan Abdelkarim Almajali, Assistant Professor of Management Information Systems, Management Information Systems Department, Faculty of Economics and Administrative Sciences, Zarqa University, Zarqa, Jordan, almjalidmaithan@yahoo.com

Dr. Ra'ed (Moh'd Taisir) Masa'deh, Associate Professor of Management Information Systems, Management Information Systems Department, School of Business, The University of Jordan, Amman, Jordan, r.masadeh@ju.edu.jo

Dr. Rand Hani Al-Dmour, Assistant Professor of Management Information Systems, Management Information Systems Department, School of Business, The University of Jordan, Rand.Aldmour@ju.edu.jo

\begin{abstract}
:
The present study aimed to develop a theoretical framework toward e-learning adoption in universities through conducting a comprehensive review of literature, and empirical studies. Based on the technology acceptance model, the researchers developed a model designed to measure the impact of ease of use, perceived usefulness, and training on e-learning usage on the actual use of e-learning systems via the initial trend towards the use of e-learning systems and the intention towards the use of e-learning systems as mediating factors. The researchers also developed a survey questionnaire that will be distributed to a selected sample study on the students at the Jordanian public and private universities in order to answer the research questions and test its hypotheses by applying structural equation modeling.
\end{abstract}

Keywords:

E-learning, technology acceptance model, theoretical study

\section{Citation:}

Al-Majali, Dr. Dmaithan Abdelkarim; Masa'deh, Dr. Ra'ed (Moh'd Taisir); Al-Dmour, Dr. Rand Hani (2016); The Role of Information Technology in motivating students to accept e-learning adoption in universities: A case study in Jordanian universities; Journal of Business \& Management (COES\&RJ-JBM) Vol.4, No.1, pp.36-46.

This work is licensed under a Creative Commons Attribution 4.0 International License. 


\title{
Journal of Business \& Management (COES\&RJ-JBM), 4(1), 36-46
}

\author{
دور تكنولوجيا المعلومات في تحفيز قبول الطلبة لبيئة التعلم الاكتروني في الجامعات : دراسة نظرية

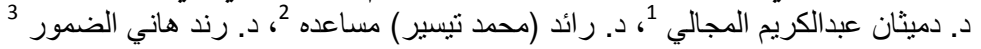 \\ قسم نظم المعلومات الإدارية، كلية الاقتصاد والعلوم الادارية، جامعة الزرقاء ، الزرقاء/الأردن، \\ almjalidmaithan@yahoo.com ${ }^{1}$ \\ قسم نظم المعلومات الإدارية، كلية الأعمال، الجامعة الأردنية، عمان/الأردن، \\ r.masadeh@ju.edu.jo ${ }^{2}$ \\ Rand.Aldmour@ju.edu.jo33
}

هدفت الدراسة الحالية الى تطوير إطار نظري لتحفيز تفاعل الطلبة في الجامعات مع بيئة التعلم الالكتروني من خلال مراجعة

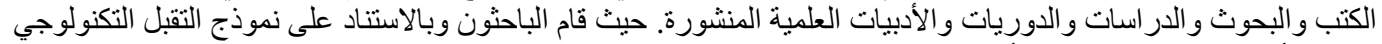

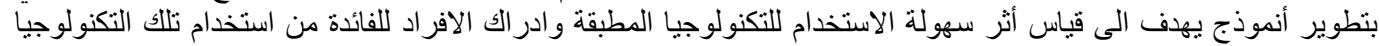

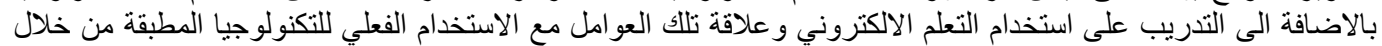

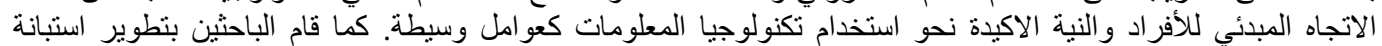

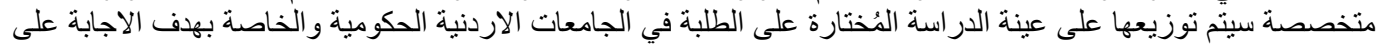
اسئلة الدراسة المقترحة واختبار فرضياتها من خلال نماذج المعادلات الهيكات الهية.

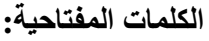
التعلم الالكتروني، نموذج التقبل التكنولوجي، دراسة نظرية.

مقدمة:

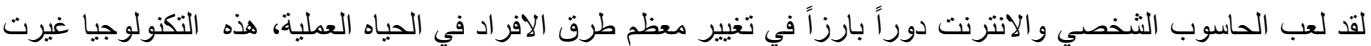

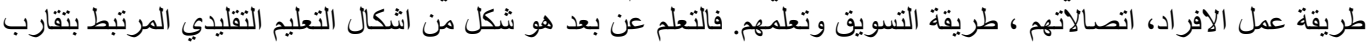

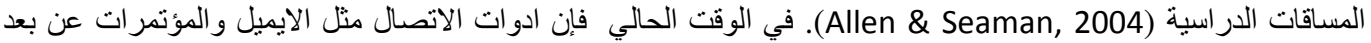

زودت المتعلمون بادو ات اتصال لجميع الطلبه في جميع انحاء العالم (Theriot, 2004; Masa'deh et al., 2013).

يتبلور مفهوم تكنولوجيا التعليم مع مرور الأيام وخصوصاً في المنطقة العربية بعد أن أدرك الددربون اهية تكامل العناصر

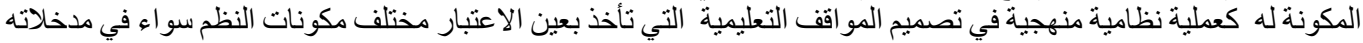

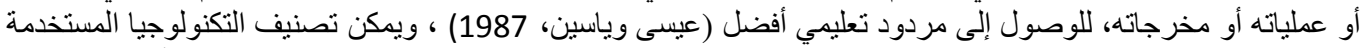

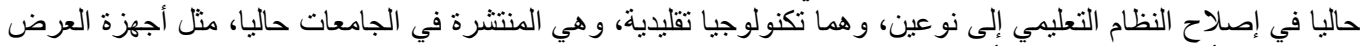

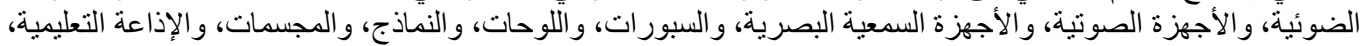

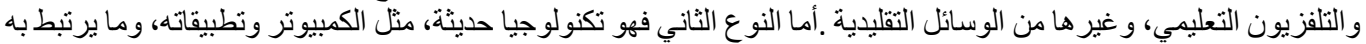
من الثبكات المحلية والعالمية مثل :الإنترنت، والبريد الالكتروني، و التعليم الافتر اضي النياني (الجملان،2004) .

وتكمن أهية التكامل التكنولوجي في تحسين نو عية التعليم و الذب أخذ حيز أ متقدمًا في بر امج التعليم منذ أو اخر عام (1980) حيث

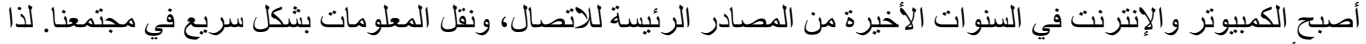

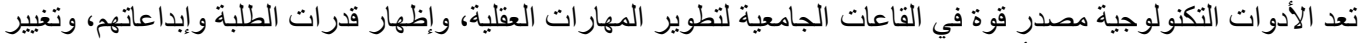

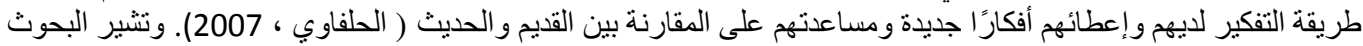

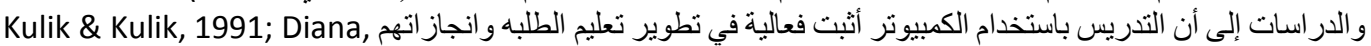

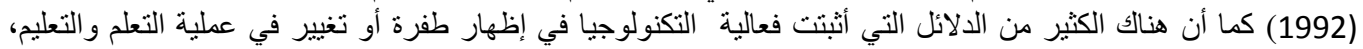

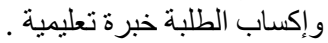

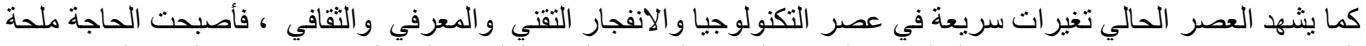

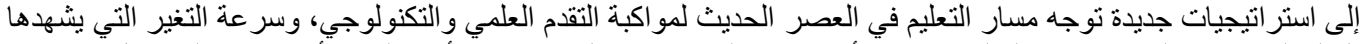

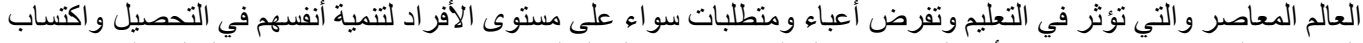

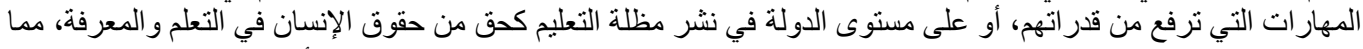

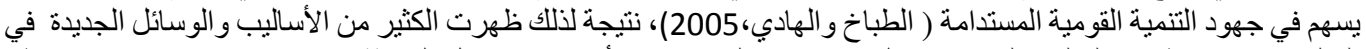

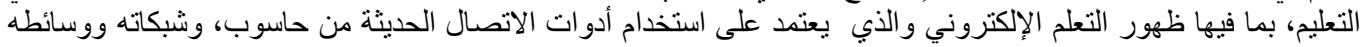


المتعددة من صوت، وصورة، ورسومات، ومكتبات الكترونية أي استخدام التقنية بجميع أشكالها؛ لإيصال المعلومة للمتعلم

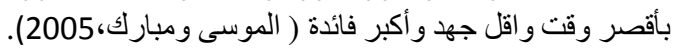

وقد أورد عبد العزيز (2008) عدداً من المميزات التي تميز التعلم الإلكتروني عن غيره من الأنواع منها :أنه يوفر التعلم في أبي

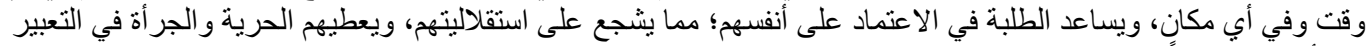

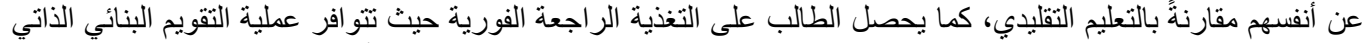

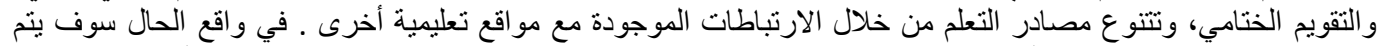

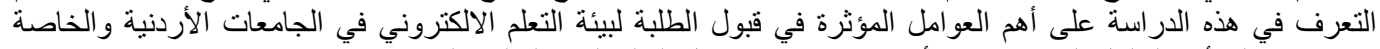

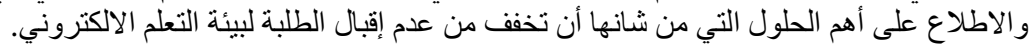

مشكلة الدراسة

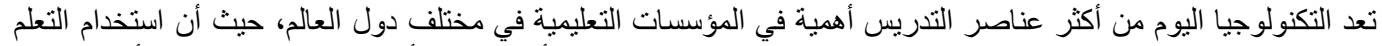

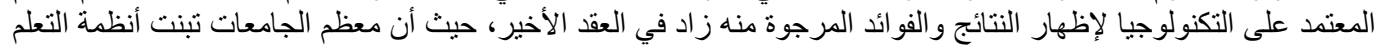
الالكتروني للمساعدة في إعطاء محتوى المساقات بشكل الكتروني (Alenezi \& Shahi, 2015).

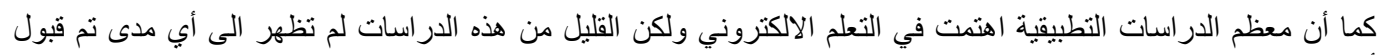

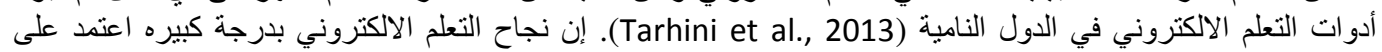

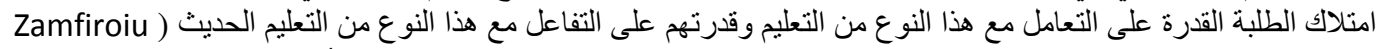

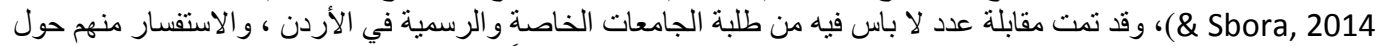

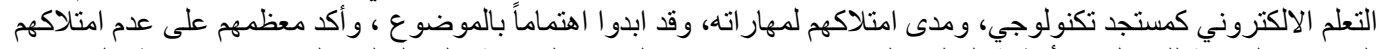

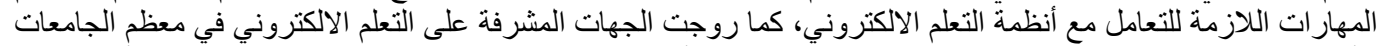

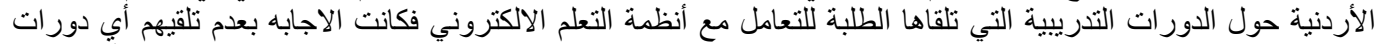

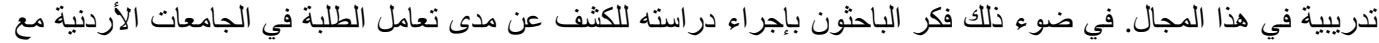
انظمه التعلم الالكتروني وما هي المعوقات التي تمنع استخدامها من قبل الطلبة في تللك الجامعات.

أسئلة الدراسة

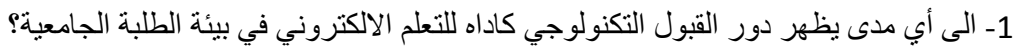

2- الى أي مدى يظهر دور الفائده من ادو ات القبول التكنولوجي مثل الانترنت، استخدام الكمبيوتر في بيئة الطلبة الجامعية؟

أهداف الاراسة 1- لتحديد الى أي مدى يمكن للقبول التكنولوجي ان يكون اداه للتعلم الالكتروني في بيئة الطلبة الجامعية. 2- لتوضيح الفائده من ادو ات القبول التكنولوجي مثل الانترنت، استخدام الكمبيونر في بيئة الطلبة الجامعية.

أهمية الاراسة تتمثل أهمية هذه الدراسة بأنها تحاول التعرف على العو امل المشجعة لاستخدام منظومة التعلم الإلكتروني التي أنشأتها معظم

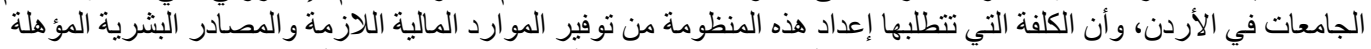

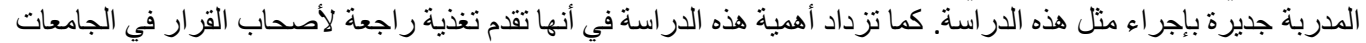

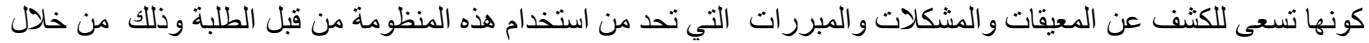

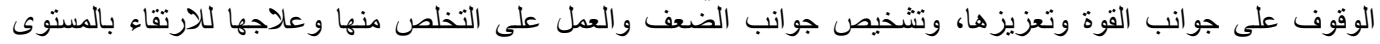
المطلوب للعملية التعليمية.

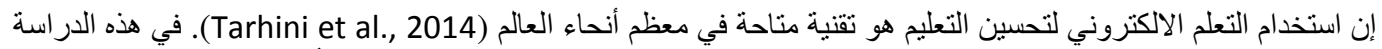

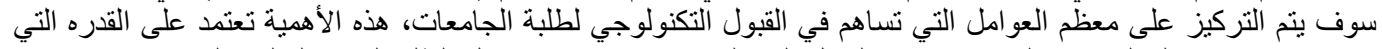

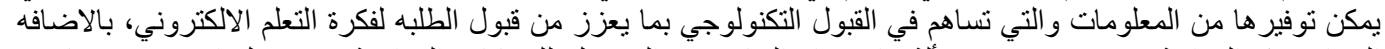

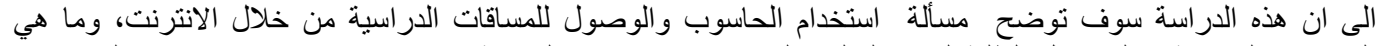

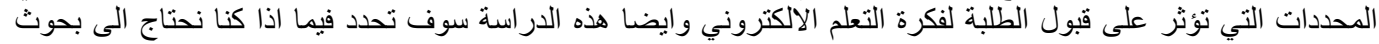
تطبيقية لمعرفة الفجوة التكنولوجية للطلبه و التي يمكن ان تؤُثر على استخدام التعلم الالكتروني.

أدبيات الدراسة 


\section{Journal of Business \& Management (COES\&RJ-JBM), 4(1), 36-46}

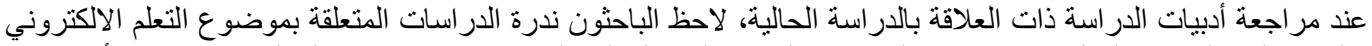

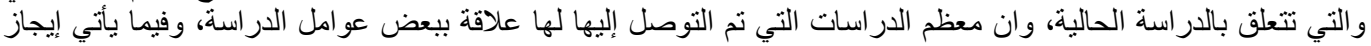

اجرى همشري وبو عزة (2000) در اسة تطبيقية للكثف عن و اقع استخدام شبكة الانترنت من قبل اعضاء هيئة التدريس بجامعة التباء

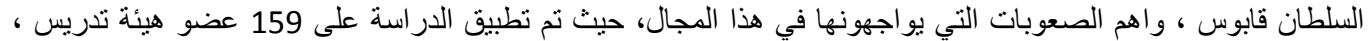

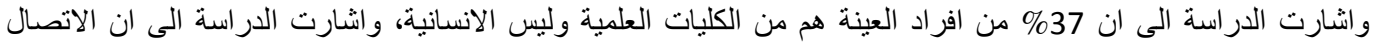

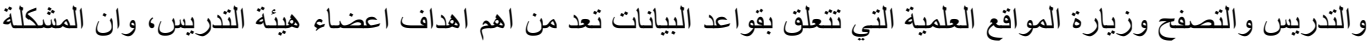

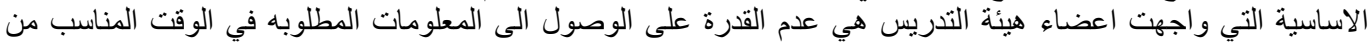

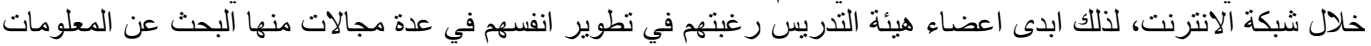

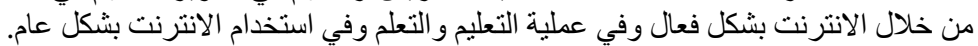

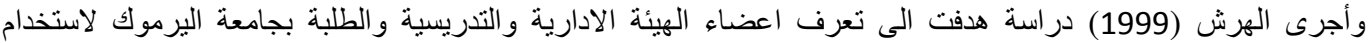

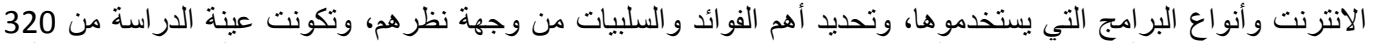

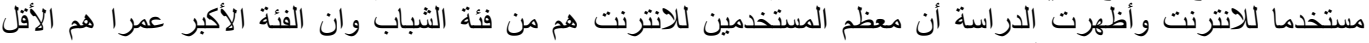

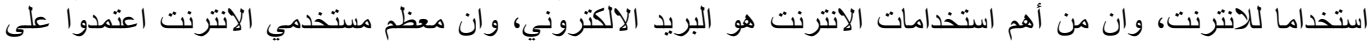

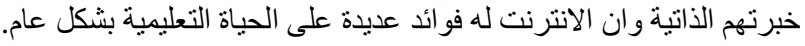

وأجرى الثايب (2001) دراسة هدفت الى التعرف على واقع استخدام أعضاء هيئة التدريس في الجامعات الأردنية

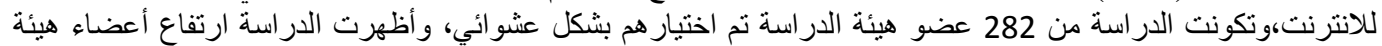

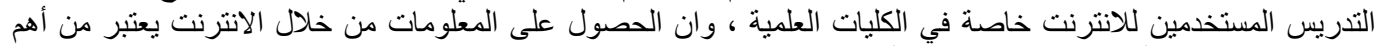

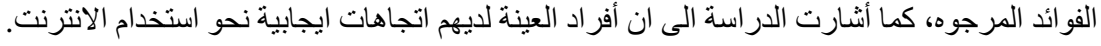

و هدفت دراسة (Allehaibi,2001) الى التعرف على واقع استخدام اعضاء هيئة التدريس للانترنت في الثتين من الجامعات

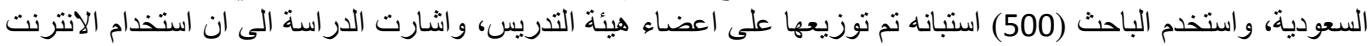

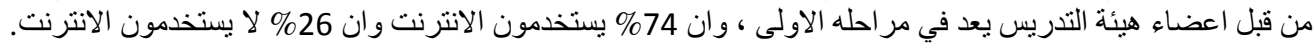

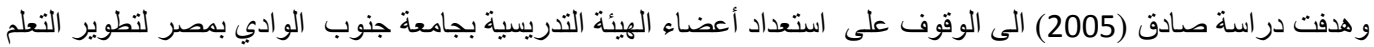

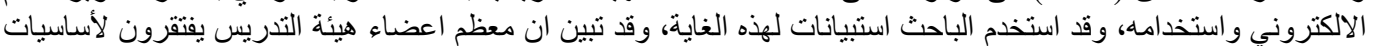

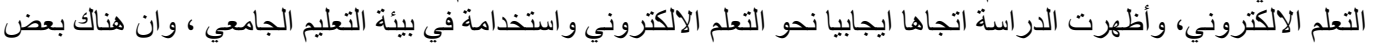

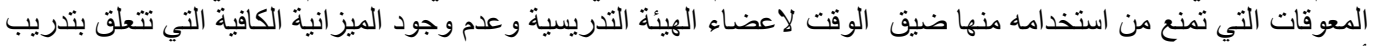
أعضاء هيئة التندريس على التعلم الالكتروني.

كما اجرى (الوشاحي، 2015) دراسة استهذف من خلالها توضيح اثر استخدام التعلم الالكتروني التعاوني في تتمية مهارات

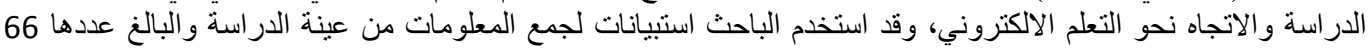

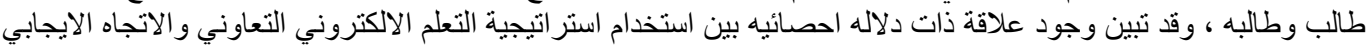

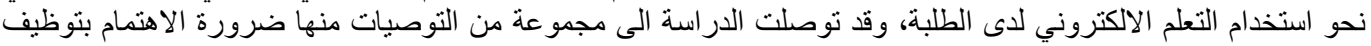

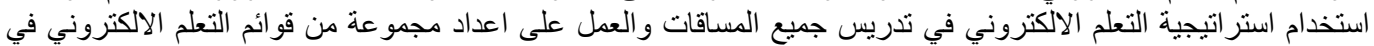
جميع المساقات الدراسية.

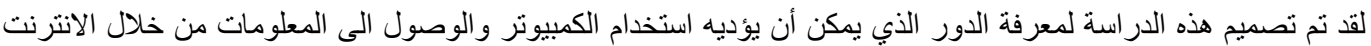

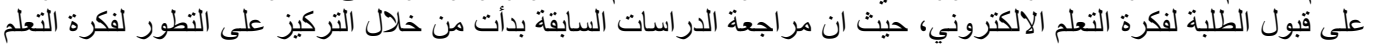

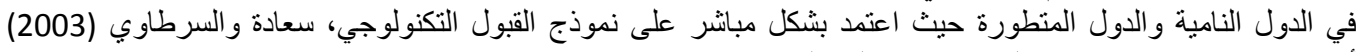
أوردوا عدة مبرر اتل لاستخدام الحاسوب كوسيلة تعليمية:

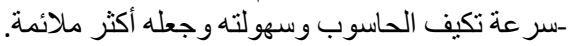

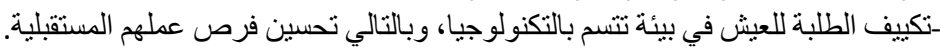

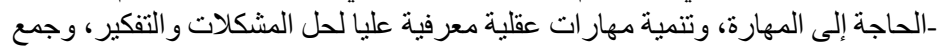

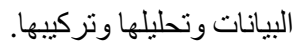


1- التعلم عن بعد: التعلم عن بعد هو عباره عن التعلم الذي يكون فيه المدرسون والمتعلمون مفصولين مكانيا وزمانيا، حيث ان

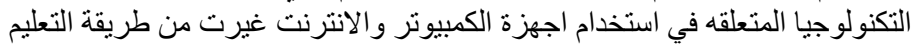

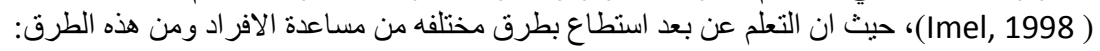

- الفيديو و المؤتمر ات عن بعد.

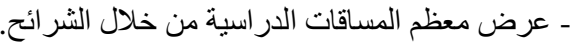

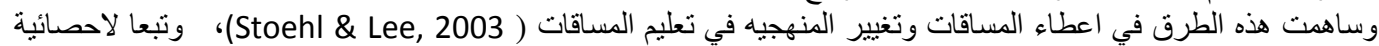

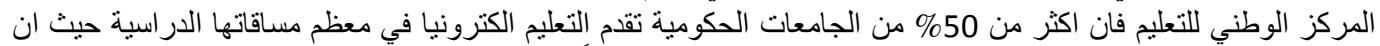

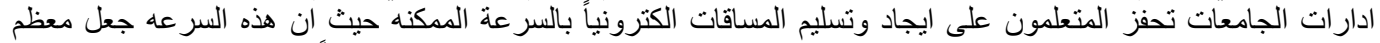

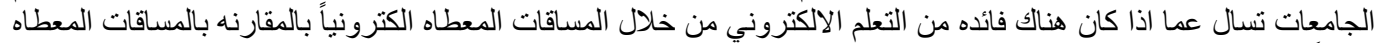
تقليدياً ام لا.

2- نموذج القبول التكنولوجي: حيث ركز هذا النموذج على الاستخدام الفعلي لتكنولوجيا المعلومات من قبل الأفراد حيث ركز

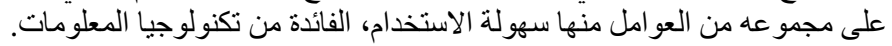

التدريب على أنظمة التعلم الالكتروني:

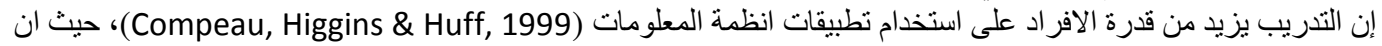

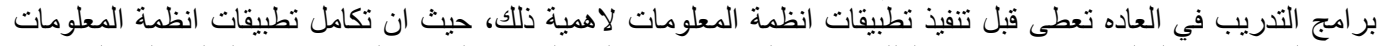

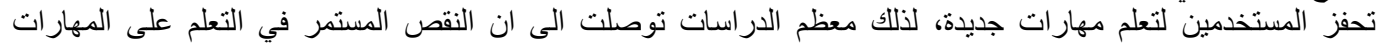
التكنولوجية الجديدة سوف يسبب فجوه بين الاستخدام الفعلي لتكنولوجيا المعلومات وبين انتون التجاه الافر اد نحو استخدام تكنولوجيا

المعلومات (Doll et al., 2003).

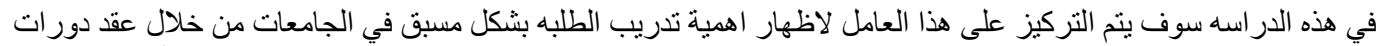

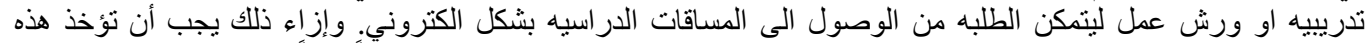

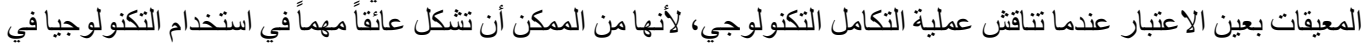

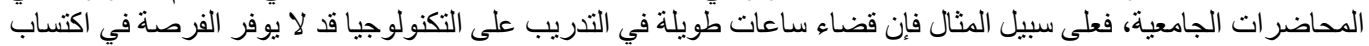

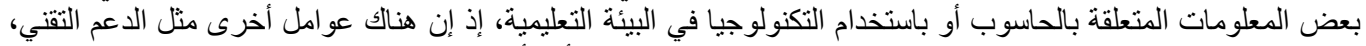

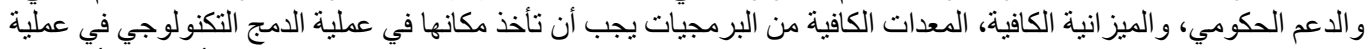

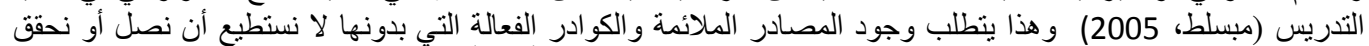

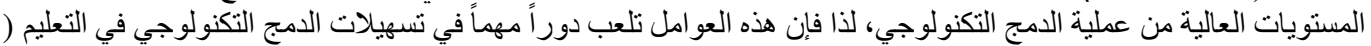

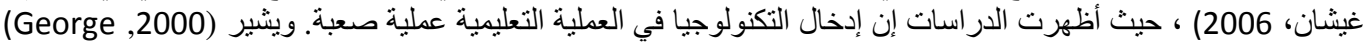

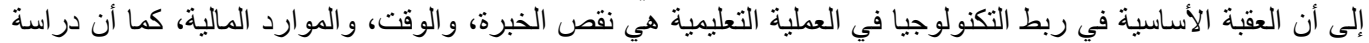

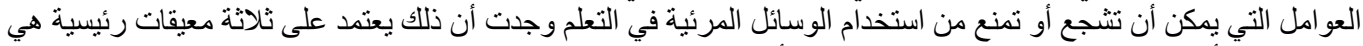

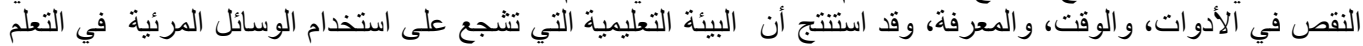

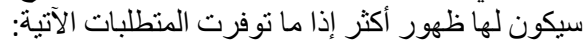

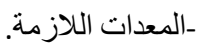

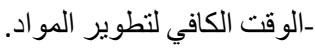
ـالاعم التقني. -سهولة استخدام البرمجيات.

كما أن الجانب المهم الآخر في عملية الدمج التكنولوجي هو القلق من استخدام التكنولوجيا، إذ منذ دخول الحاسوب في البيئة

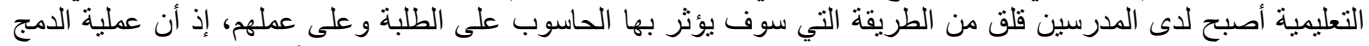

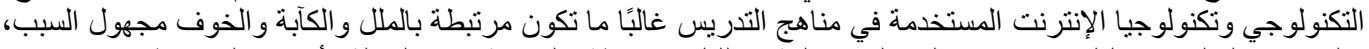

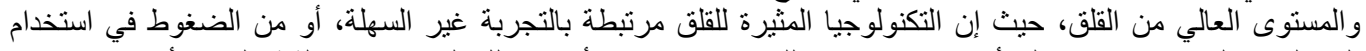

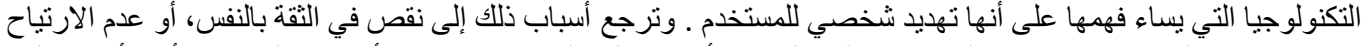

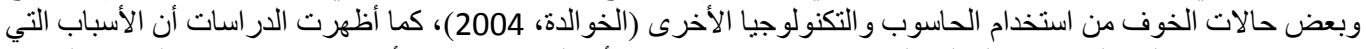

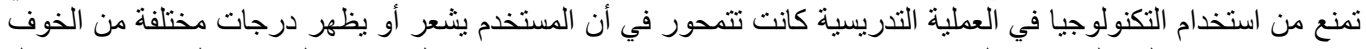

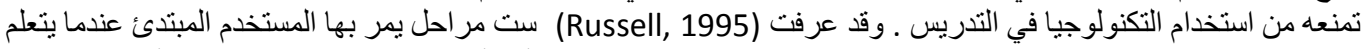

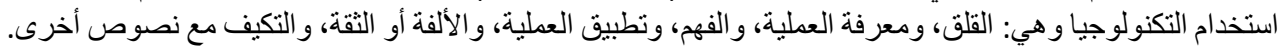




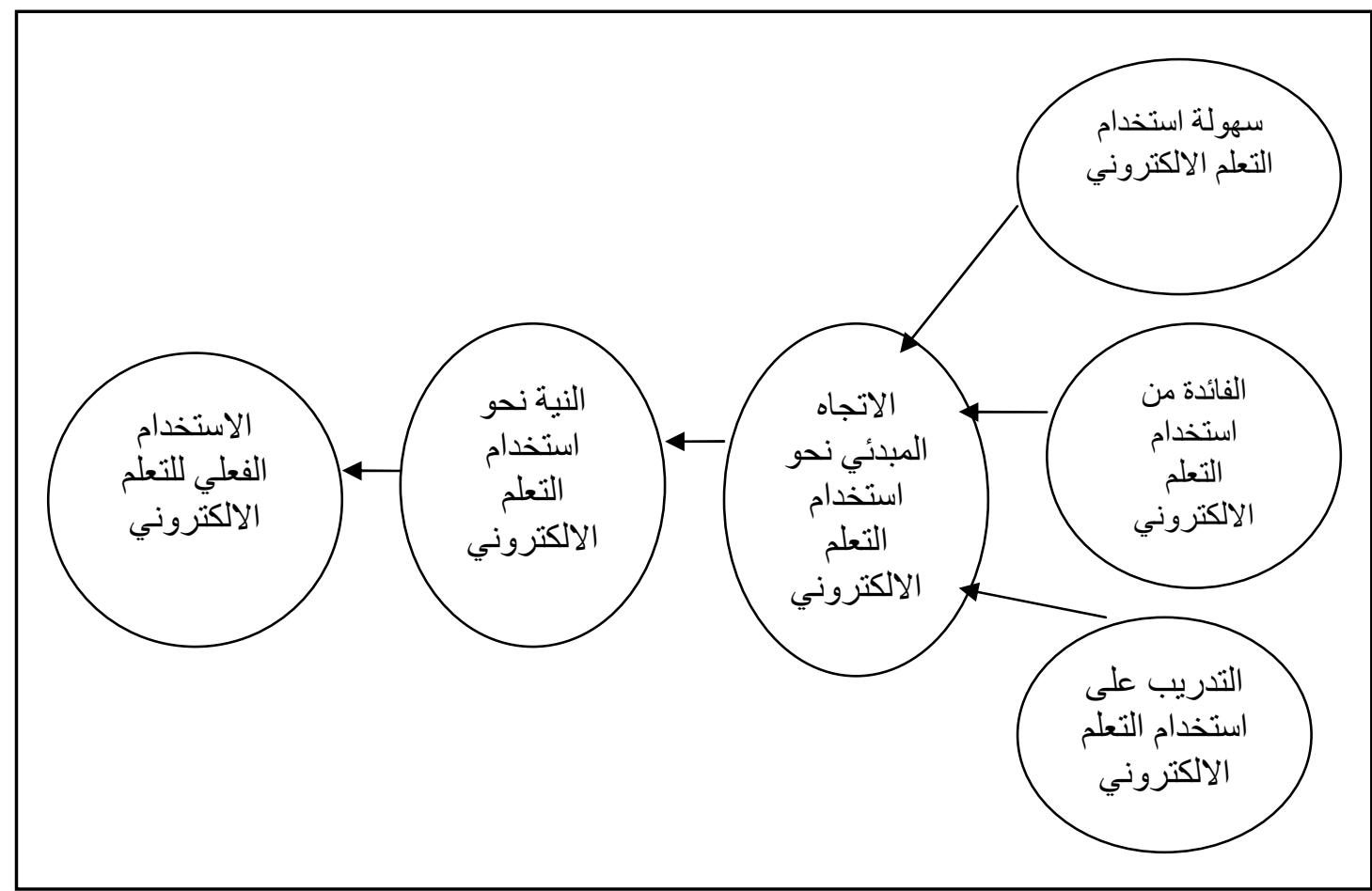

الثكل 1. نموذج الاراسة

في هذه الدر اسة تم إعداد مجمو عة من العبارات لقياس متغير ات الدراسة كما هو مبين في الجدول حيث تم الاعتماد على مجمو عه من الدراسات مع إجراء بعض التعديلات على نلآلك العبار ات.

جدول 1. متغيرات الاراسة وعباراتها

\begin{tabular}{|c|c|}
\hline 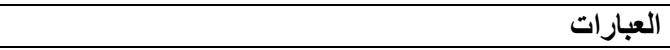 & 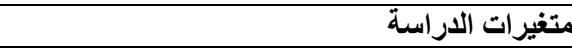 \\
\hline التعلم الالكتروني سهل الاستخدام & \multirow{5}{*}{ 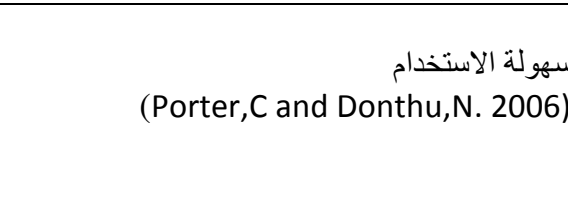 } \\
\hline التعلم الالكتروني و اضح ومفهوم & \\
\hline التعلم الاككتروني سهل ويمكن الطلبة من استخر اج المعلومات & \\
\hline 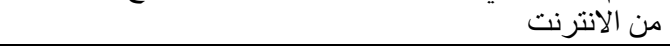 & \\
\hline على جميع الأحوال، التعلم الالكتروني سهل الاستخدام & \\
\hline بساعد استخدام أنظمة التعلم الاكتكروني على زيادة الإنتاجية & \multirow{4}{*}{$\begin{array}{r}\text { الفائدة من الاستخدام (Porter,C and Donthu,N. 2006) } \\
\text { (Ponthe }\end{array}$} \\
\hline إن استخدام أنظمة التعلم الالكتروني يجعل الوصول الىى & \\
\hline المساقات أسهل & \\
\hline على جميع الأحوال، استخدام أنظمة التعلم الالكتروني مفيد & \\
\hline يتم تحديد الاحتياجات التدريبية في الجامعة على نحو علمي & \multirow{7}{*}{ (الغامديب، عالد استخدام أنظمة التعلم الالكتروني } \\
\hline وبما يحقق أهداف التدريب & \\
\hline تتحدد احتياجات التدريب في ضوء الاحتياجات الفعلية للطلبة & \\
\hline اخضع لدور ات تدرييية تساعدني على حل الشكلات & \\
\hline التدريبية بصوره أفضل & \\
\hline ان التدريب سوف يؤدي الى إحداث تطوير في أدائي & \\
\hline ينتم متابعتي بعد انتهاء فترة التدريب & \\
\hline انا ايجابي اتجاه استخدام أنظمة التعلم الالكتروني & \multirow[b]{2}{*}{ الاتجاه المبدئي نحو استخدام انظمة التعلم الالكتروني } \\
\hline بشكل أفضدل إنظمة التعلم الالكتروني تجعل المساقات مفهومه & \\
\hline
\end{tabular}


Journal of Business \& Management (COES\&RJ-JBM), 4(1), 36-46

\begin{tabular}{|c|c|}
\hline يجب ان أتبنى أنظمة التعلم الالكتروني & (Porter,C and Donthu,N. 2006) \\
\hline لذلك عندي النتية الاكيده نحو استخدول التى أنظمة التعلم الالكتروني & \multirow[b]{2}{*}{$\begin{array}{l}\text { النية نحو استخدام أنظمة التعلم الالكتروني (Davis,1989)، (Willis , T. 2008) } \\
\text { (Dis 2008) }\end{array}$} \\
\hline 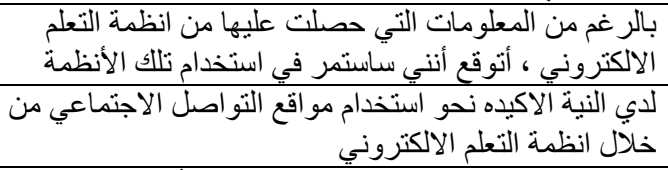 & \\
\hline شخصية تعليمية التعلم الالكتروني في بعض الأحبان لفو ائد & \multirow[t]{2}{*}{$\begin{array}{l}\text { الاستخدام الفعلي لأنظمة التعلم الالكتروني } \\
\text { (Porter,C and Donthu,N. 2006) }\end{array}$} \\
\hline 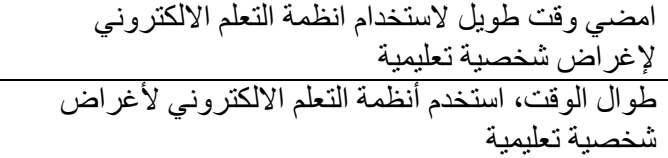 & \\
\hline
\end{tabular}

الفرضيه الاولى: يوجدئ علاقة ذات دلاله احصائيه بين سهولة الاستخدام للتعلم الالكتروني والاتجاه المبدئي نحو استخدام التعلم الالكتروني

الفرضيه الثانية: يوجد علاقه ذات دلاله احصائيه بين الفائدة من استخدام التعلم الاكتروني والاتجاه المبدئي نحو استخدام التعلم

الالكتروني

الفرضيه الثالثة: يوجد علاقة ذات دلاله احصائيه بين التدريب على استخدام التعلم الالكتروني والاتجاه المبئي نحو استخدام التعلم الالكتروني

الفرضيه الر ابعة: يوجد علاقه ذات دلاله احصائيه بين الاتجاه المبدئي نحو استخدام التعلم الالكتروني والنية نحو استخدام التعلم الالكتروني الفزضن الري

الفرضيه الخامسة: يوجد علاقه ذات دلاله احصائيه بين النية نحو استخدام التعلم الالكتروني و الاستخدام الفعلي للتعلم الالكتروني

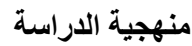

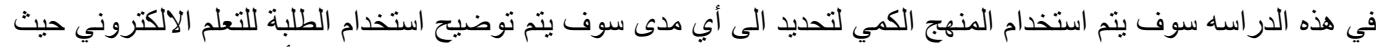

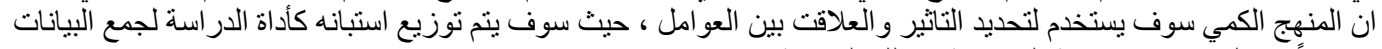

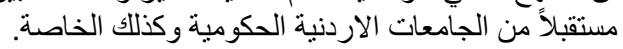

مجتمع الدراسة

سيتكون مجتمع الدر اسة من طلبة الجية الجامعات الاردنية الحكومية والخاصة، اما عينة الدراسة سوف يتم اختيار ها بشكل عشو ائي

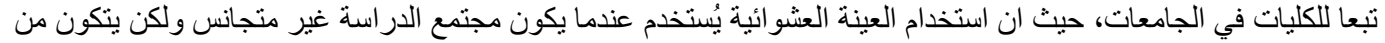
عدة مجتمعات فر عية افية (Wiersma, 2000).

النتائج والتوصيات

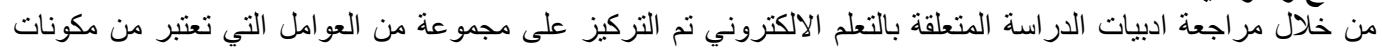

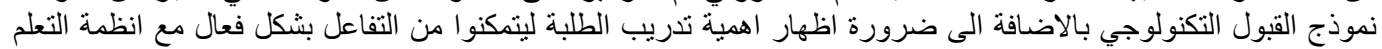

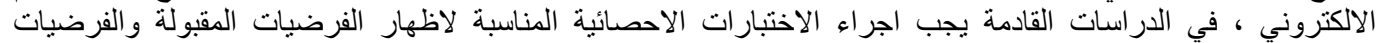

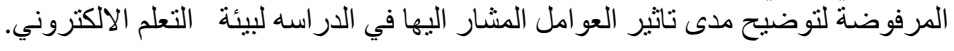


في هذا البحث ثم التحقق من ادبيات التعلم الالكتروني و التوصل التى اهم العو امل التي يمكن ان تؤئر في قبول الطلبة لبيئة التعلم

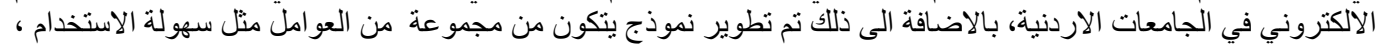

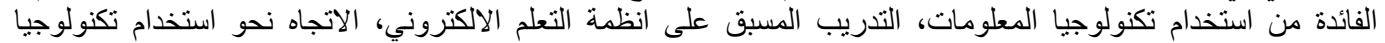

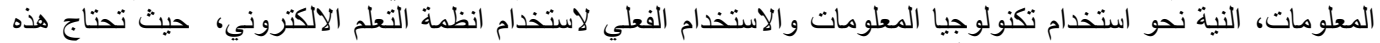

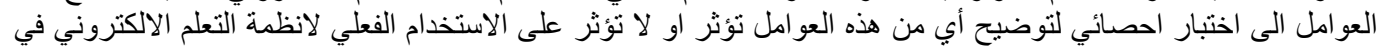

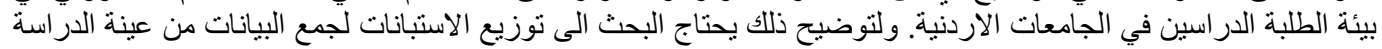

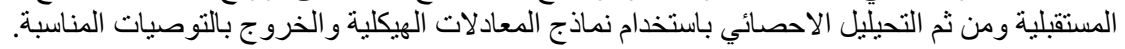

\section{References}

المراجع المع اجع

المراجع الاجنبية

Alenezi, A., and Shahi, K. (2015). Interactive e-learning through second life with blackboard technology. Procedia Social and Behavioral Sciences, 176, 891-897.

Allehaibi, M. (2001). Faculty adoption of Internet technology in Saudi Arabian universities. PhD, Florida State University. Available: http://www.sssgrp.com/Menu/Di ssAbstracts /InnovationDiffusion/Allehaibi.htm. Retrieved on 12/4/2015.

Allen, I., and Seaman, J. (2004). Entering the mainstream: The quality and extent of online education in the United States, 2003 and 2004. Retrieved April 15, 2005 from: http://sloanc.org/resources/entering mainstream.pdf.

Clark, Jr. T.D., Jones, M.C., and Zmud, R.W. (2006). Post adoptive ERP system analysis: A system dynamic modeling approach, Working Paper, http://www.bus.Isu.edu./centers/decid/WorkingPaper.asp.

Compeau, D.R., Higgins, C.A., and Huff, S.L. (1999). Social cognitive theory and individual reactions to computing technology: A longitudinal study. MIS Quarterly, 23(2), 145-158.

Davis, F. D. (1989). Perceived usefulness, perceived ease of use, and user acceptance of information technology. MIS Quarterly, 13(3), 319-340.

Davis, F., Bagozzi, R., and Warshaw, P. (1989). User acceptance of computer technology: A comparison of two theoretical models. Management Science, 35(8), 982-1003.

Diana, O. (1992). Teaching and learning with computers. (URL: $\underline{\text { http://orders.edrs.com). }}$

Doll, W.J., Deng, X., and Scazzero, J.A. (2003). A process for post-implementation IT benchmarking, Information \& Management, 41, 199-212.

George, P. (2000). Breaking ranks. Principal Leadership, 1(4), 56-61.

Imel, S. (1998). Myths and realities. (Report No. BBB16032). Columbus, OH. ERICClearinghouse on Adult, Career, and Vocational Education.

Kulik, J. A., and Kulik, C. L. (1991). Effectiveness of computer-based instruction. Computers in Human Behavior, 7(1), 75-94. 
Masa'deh, R., Shannak, R., and Maqableh, M. (2013). A structural equation modeling approach for determining antecedents and outcomes of students' attitude toward mobile commerce adoption. Life Science Journal, 10(4), 2321-2333.

Porter,C and Donthu,N. (2006) . Using the technology acceptance model to explain how attitudes determine Internet usage: The role of perceived access barriers and demographics. Journal of Business Research, 59, 999-1007.

Russell, A. I. (1995). Stages in learning new technology: Naïve adult e-mail users. Computers in Education, 25(4), 173-178.

Stoehl, L., and Lee, K. (2003). Modeling the effect of experience on student acceptance of Webbased courseware. Internet Research: Electronic Networking Applications and Policy, 13(5), 364374.

Tarhini, A., Hone, K., and Liu, X. (2013). Factors affecting students' acceptance of e-learning environments in developing countries: A structural equation modelling approach. International Journal of Information and Education Technology, 3(1), 54-59.

Tarhini, A., Hone, K., and Liu, X. (2014). Measuring the moderating effect of gender and age on e-learning acceptance in England: A structural equation modeling approach for an extended technology acceptance model. Journal of Educational Computing Research, 51(2), 163-184.

Theriot, P. (2004). Student values and ethics in an e-learning environment. In D. Christopher \& S. Jaderstrom (Eds.), NBEA 2004 Yearbook, 42, 13-25.

Wiersma, W. (2000). Research methods in education: An introduction (7nd ed.). Boston:Allyn and Bacon.

Willis, T (2008). An evaluation of the Technology Acceptance Model as a means of understanding online social networking behaviour, Master thesis, University of South Florida.

Zamfiroiu, A., and Sbora, C. (2014). Statistical analysis of the behavior for mobile E-learning. Procedia Economics and Finance, 10, 237-243.

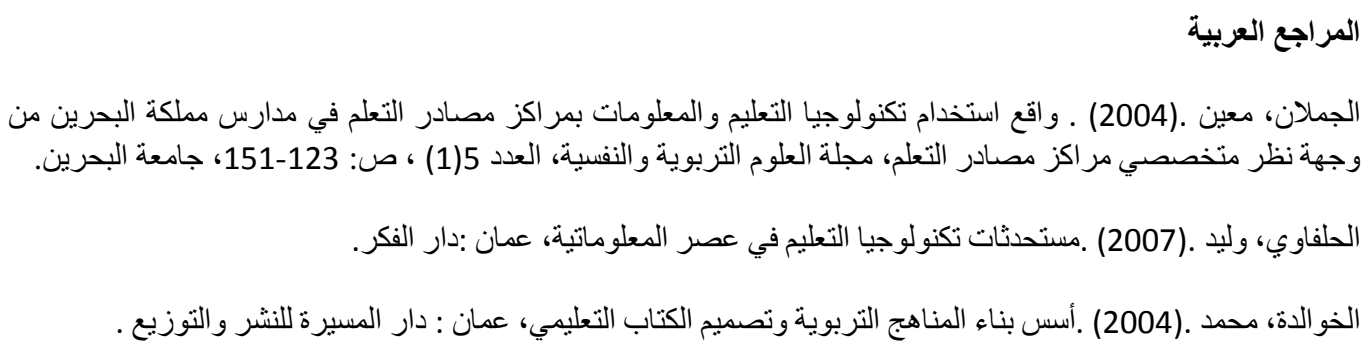


الثايب، احمد. (2001). و اقع استخدام اعضاء هيئة التدريس في الجامعات الاردنية لشبكة الانترنت و اتجاهاتهم نحوها، رسالة ماجستير غير منشوره ، جامعة اليرموك، اركب، اعضد، الاردن.

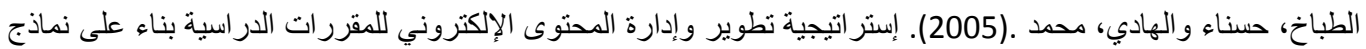

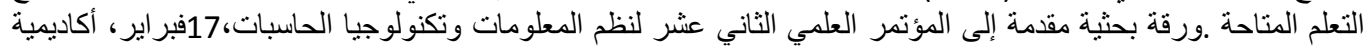
السادات للعلوم الإدارية، القاهرة، 15.

الغامدي، خالد. (2010). دور التدريب في رفع كفاءة اداء موظفي القطاع العام. دراسة حالة وزارة الثؤون الاجتماعية ـ

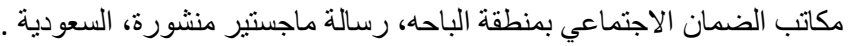
الموسى ، عبدالله و المبارك،احمد. (2005). التعلم الالكتروني: الاسس و التطبيقات. الرياض: دار العبيكان.

الهرش ، عايد حمدان. ( 1999). استخدام شبكة الانترنت من قبل اعضاء هيئة التدريس و الطلبة و الموظفين و الاداريين بجامعة

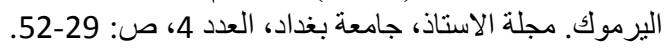

الوشاحي ،عمار ـ (2015). اثر استخدام إستر اتيجية التعلم الالكتروني التعاوني في تتمية مهار ات الدراسة التهات والاتجاه نحو التعلم

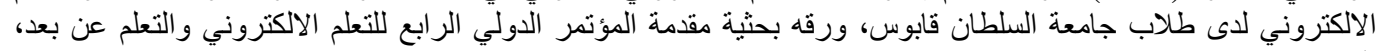

سعاده، جودت و السرطاوي، عادل ـ(2003). استخدام الحاسوب و الانترنت في ميادين التربية والتعليم، دار الثروق للنشر و التوزيع.

صادق، علاء (2005). استعداد اعضاء هيئة التدريس بجامعة جنوب التوادئ التوادي لتطوير واستخدام التعلم الالكتروني. الجمعية

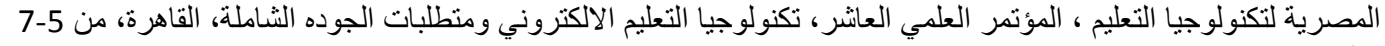
يوليو. عبد العزيز، حمدي .(2008). التعليم الإكتروني الفلسفةـ المبادئـ الأدواتـ التطبيقات، عمان :دار الفكر. عيسى، مصباح وياسين، عادل .(1987) ، التقنيات التربوية في تدريس الرياضيات بالمرحلة الابتدائية، مجلة العلوم الاجتماعية ، .254-233 ، (2)15

غيشان، ريما .(2006) ـ درجة اهتمام معلمي المرحلة الأساسية العليا (الصف العانشر) في المدارس الحكومية في مديريات تربية محافظة العاصمة بتكنولوجيا التعليم واتجاهات الطلبة نحوها، أطروحة دكتور اه غير منشورة، الجامعة الأردنية، عمان، الأردن.

مبسلط، ملك .(2005 ). و اقع استخدام معلمي المرحلة الثانوية لتكنولوجيا المعلومات والاتصالات في التدريس في المدارس الثانوية الحكومية في عمان، رسالة ماجستير غير منشورة، الجامعة الأردنية، عمان، الأردن.

همشري، عمر وبو عزة، عبد المجيد (2000). واقع استخدام شبكة الانترنت من قبل اعضاء هيئة التدريس بجامعة السلطان قابوس. مجلة در اسات، الاردن، العدد 27(2)، صع: صند: 328-342.

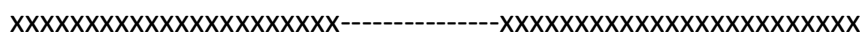

